\title{
LA RELACIÓN ENTRE LA ANTROPOLOGÍA MEXICANA Y ECUATORIANA: ¿UN CAMINO DE IDA Y VUELTA?
}

The Relationship between Mexican and Ecuadorian Anthropology: ¿A Round Trip Journey? FERNANDO GARCÍA S. *

\section{Resumen}

Este trabajo consiste en una revisión del desarrollo de la antropología mexicana y ecuatoriana desde la perspectiva de los diálogos que atraviesan las propuestas teóricas y metodológicas, considerando desde la formación de profesionales hasta la puesta en marcha de políticas públicas relacionadas con la llamada "antropología aplicada". Mediante un esbozo rápido y general de seis décadas del naciente pensamiento antropológico ecuatoriano -aunque sin que este sea un balance- se busca establecer cómo la interrelación entre antropología mexicana y ecuatoriana, muestra un camino de ida y vuelta, construido por el cruce de diversas perspectivas teóricas generadas al interior del debate disciplinar con los énfasis e intereses diferenciados de los actores locales.

Palabras Clave: Antropología, México, Ecuador

\section{Abstract}

This paper is a review of the development of Mexican and Ecuadorian anthropology from the perspective of the dialogues that cross the theoretical and methodological issues, considering since the training of professionals until the implementation of public policies related to the call "applied anthropology." Through a rapid and general outline of six decades of nascent Ecuadorian anthropological thought - which is not pretending to be a final balance-, it looks to establish how the relationship between Mexican and Ecuadorian anthropology shows a way back and forth, built by the crossing of different theoretical perspectives generated into the discipline discussion with the emphasis and differentiated interests of local actors.

Key words: Anthropology, México, Ecuador

\footnotetext{
* Antropólogo. Profesor Investigador FLACSO-Ecuador. Miembro de la Red Latinoamericana de Antropología Jurídica (RELAJU). Correo electrónico: fgarcia@flacso.edu.ec
} 


\section{Introducción}

Los vínculos interculturales que relacionaron las realidades mexicanas y ecuatorianas desde inicios del siglo $\mathrm{XX}$, no solo se centraron en las expresiones culturales en general, sino en otros aspectos relacionados también con la cultura, comprendida más desde la perspectiva disciplinar, como es el caso de la antropología definida como "ciencia social interesada" como menciona Geertz (1988), "en una labor de interpretación en busca de significados".

La revisión del desarrollo de la antropología mexicana y ecuatoriana muestra un conjunto de diálogos mutuos que van desde las propuestas teóricas y metodológicas, pasando por la formación de profesionales, hasta la puesta en marcha de políticas públicas relacionadas con la llamada "antropología aplicada". Esta elaboración se inspira en una corriente iniciada por un conjunto de antropólogos provenientes de lugares considerados periféricos, entre ellos, los latinoamericanos, que están empeñados en pensar en las denominadas "antropologías del mundo" (Ribeiro \& Escobar, 2006) y no en una antropología universal, como un forma de recuperar uno de los ejes sobre los cuales ha girado la antropología como ciencia, la diversidad científica y conceptual.

Mediante un esbozo rápido y general de seis décadas del naciente pensamiento antropológico ecuatoriano, que de ninguna manera intenta ser un balance de la disciplina, pretendo desarrollar el argumento que la interrelación entre dos "antropologías del sur" (Krotz, 1997) o periféricas (Cardoso, 1998), la mexicana y ecuatoriana, muestra un camino de ida y vuelta construido por el cruce de diversas perspectivas teóricas generadas al interior del debate disciplinar, con los énfasis e intereses sociales de los actores locales (Jimeno, 2007).

Con este objeto, propongo diferenciar tres grandes etapas durante el período 1910-1970, que permitan distinguir los principales quiebres que separan a uno de otro. El primero, se inicia con los aportes de los primeros indigenistas mexicanos a inicios de siglo, continúa con la aprobación de la legislación social adoptada por Ecuador en la década de 1930 a 1940 y culmina con la convocatoria al I Congreso Indigenista Interamericano en 1940 en Pátzcuaro, Michoacán, y la subsiguiente creación del Instituto Indigenista Ecuatoriano.

El segundo, comprende la puesta en marcha de la política conocida como el "desarrollo de la comunidad", considerada una segunda fase del indigenismo, representada en el Ecuador por la ejecución del programa denominado Misión Andina (1954-1972), así como el inicio de la formación de los primeros antropólogos ecuatorianos en México. El último período arranca con la crisis del indigenismo y el surgimiento de la nueva antropología mexicana, a partir de la declaración de Barbados I y II, la continuidad en la formación de una segunda generación de antropólogos ecuatorianos y, el énfasis en los estudios sobre diversidad cultural y étnica, como también de los estudios etnohistóricos comparativos, entre los Andes y Mesoamérica.

\section{La propuesta indigenista}

El particularismo histórico o relativismo cultural, planteado por Franz Boas como corriente de pensamiento dentro de la antropología a inicios del siglo $\mathrm{XX}$, especialmente desde su cátedra de la Universidad de Columbia en New York, sostenía que no se debía hablar de cultura en singular, 
sino de culturas en plural ya que todas ellas eran diferentes y que no podían medirse con un patrón único de desarrollo.

Esta propuesta, surgida como respuesta al darwinismo social y al racismo de fines del siglo $\mathrm{XIX}$, establecía que las diferencias culturales no se explicaban por razones de pertenencia racial o por herencia biológica, sino por una singular visión del mundo que posee cada cultura. Igualmente importante es el aporte de Boas y sus discípulos norteamericanos con la incorporación de la práctica de "trabajo de campo", que van a instaurar en sus diversas investigaciones ${ }^{1}$.

Se debe reconocer la importante influencia de la propuesta "boasiana" en la antropología de entreguerras y, en el caso de América Latina, es importante examinar su presencia por lo menos en dos sentidos. El primero, en la formación de los primeros antropólogos mexicanos en la Universidad de Columbia, en especial de Manuel Gamio, uno de los propulsores del pensamiento indigenista junto con Alfonso Caso en la segunda década del siglo XX. El segundo, en la importancia de su propuesta metodológica al propiciar un énfasis paralelo entre observador y observado en la construcción etnográfica y, que es motivo de reflexión actual en las denominadas auto-etnografías (Ramos, 2007) o etnografía colaborativa (Rappaport, 2007).

El aporte de Gamio y Caso pone en debate el tema del indio en la construcción de la nación; plantean que la solución a la cuestión nacional no se resuelve solamente por la amalgama de razas sino por la mezcla de culturas (la india y la no india), que son vistas como complementarias, sujetas de interpenetración e intercambio para reducir poco a poco sus diferencias, hasta el momento que formen una sola y misma cultura ${ }^{2}$.
Se trata de un pensamiento indigenista-culturalista que nace históricamente ligado a la revolución mexicana de 1910, pero que no acaba de romper sus lazos con el positivismo del siglo anterior, al mantener al mestizaje como alternativa, aunque su concepción se modifica. No hay que olvidar que la propuesta indigenista va a ejercer una influencia determinante en la concepción, formulación y ejecución de políticas públicas referidas a los pueblos indígenas desde las Naciones Unidas, los organismos interamericanos y los gobiernos nacionales hasta la década de los setenta.

El pensamiento indigenista, desde su concepción y luego práctica, no fue de ninguna manera la manifestación del pensamiento de los pueblos indígenas, sino una reflexión de los no indígenas (mestizos y criollos) sobre el indio. En este sentido, el desarrollo del pensamiento indigenistaculturalista estuvo acompañado del pensamiento indigenista marxista, cuyos principales pensadores van a surgir en México y Perú.

El marxismo latinoamericano, antes de la revolución rusa de 1917, e influenciado por la ideología del socialismo utópico y del anarco sindicalismo, consideraba al indio como un componente indiferenciado de un "pueblo" o de un "proletariado" igualmente oprimido, sin reconocer ningún carácter específico a su situación. El escritor y político peruano, Manuel González Prada, fue quien planteó en su obra Nuestros Indios (1904), que el problema del indio no es un problema racial, tampoco es un problema cultural que encuentre su posible solución en la educación. Para él se trataba de un problema esencialmente económico y social. Esta posición va a ser retomada por otros dos autores peruanos muy influyentes en su época: José Carlos Mariátegui e Hildebrando Castro Pozo, que en sus obras los 
Siete ensayos de interpretación de la realidad peruana (1928) y Nuestra comunidad indígena (1924), van a incorporar dos temas de trascendencia en la antropología latinoamericana, el de la sobrevivencia de la comunidad indígena o ayllu y el concepto de nacionalidad indígena.

Desde México, Vicente Lombardo Toledano luego de su visita a la Unión Soviética, regresa impresionado por la manera como se resuelve allá el problema de las nacionalidades, e igualmente, las compara con las nacionalidades indígenas en su libro Un viaje al mundo del porvenir (1936), en el que plantea que se debe crear grandes centros industriales en las áreas indígenas para explotar los recursos locales, lo cual arrancaría a los indios del campo, los proletarizaría y despertaría su conciencia revolucionaria. En otras palabras, a pesar de las evidencias de la realidad mexicana se niega a definir a México como Estado plurinacional.

Hacia finales de los años veinte, la Tercera Internacional trata de orientar a los partidos comunistas latinoamericanos en los temas indígenas. Así, en la Conferencia Comunista Latinoamericana celebrada en Buenos Aires en 1929, se examina si en la lucha antiimperialista se debe incluir a las nacionalidades indígenas y la manera de hacerlo. Los dirigentes del entonces Komintern, nacionalistas en alto grado, consideran que el socialismo es el medio más rápido y seguro de construir la nación y, por lo tanto, consideran que el reconocimiento de las nacionalidades indias, retomando la tesis planteadas por Radek y Rosa Luxemburgo, desembocaría en la creación de Estados burgueses que retrasarían la revolución.

Ante estos antecedentes, es indispensable volver la mirada a la realidad ecuatoriana y analizar la influencia del pensamiento indigenista, culturalista y marxista en las condiciones locales y, en las discusiones antropológicas. Moreno (2006), al hablar del pensamiento antropológico ecuatoriano, distingue tres grandes períodos en su desarrollo. El primero, representado por los precursores ilustrados y románticos del siglo XVIII y XIX, el segundo, personificado por los antropólogos autodidactas cercanos al positivismo sociológico, al pensamiento indigenista y al difusionismo, que hicieron sus aportes durante las siete primeras décadas del siglo $\mathrm{XX}$ y, finalmente, el período llamado por Escobar (1996) para el caso colombiano, como el de la profesionalización e institucionalización de la Antropología, que empieza en 1971 con la creación del departamento de Antropología en la Universidad Católica del Ecuador.

En esta elaboración, me concentraré principalmente en el segundo período y en los inicios del tercero. También es importante contextualizar el contexto político e ideológico que vivía el Ecuador a fines del siglo XIX e inicios del XX. El hecho más significativo es la emergencia del liberalismo, similar al caso mexicano que se inició años antes, con la victoria de Benito Juárez contra el emperador Maximiliano en 1867, que suscitó en el país una lucha armada y luego, una lucha por la hegemonía ideológica y económica.

Las conquistas más importantes de la revolución liberal, están representadas por el quiebre del predominio político del partido conservador y del clero, la implantación de la educación laica, la libertad de expresión y de cultos y, la democratización de la cultura. En ese período surge con fuerza la clase media, ausente hasta entonces de la vida nacional, cuyo sector más ilustrado va a producir los principales ideólogos del liberalismo como doctrina, ya que difícilmente se puede hablar de la presencia de una burguesía local, en el sentido del liberalismo europeo, que lidere el proceso de su conformación. 
El positivismo sociológico tuvo en Ecuador a dos escritores representativos: Belisario Quevedo y Alfredo Espinosa Tamayo. Este último, con su libro la Psicología y sociología del pueblo ecuatoriano (1918), que constituye la primera obra de la sociología ecuatoriana, muestra las influencias de los padres de la sociología francesa e inglesa, Comte y Spencer, comparable a otras similares en América Latina, Bunge en Argentina, Arguedas en Bolivia y Bulnes en México. Para Espinosa Tamayo:

"la sociedad se divide en clases que se diferencian por el grado de desarrollo mental, por un lado, los hombres materiales que por su escasa mentalidad no son capaces de captar ideales elevados, el caso del indígena, y el hombre superior, en el que la idea se da junto con una voluntad ajena a entusiasmos pasajeros, una fuerza interior que le impide un ejercicio espasmódico de la misma" (Roig, 1979:116).

Respecto al pensamiento indigenista, culturalista y marxista, el iniciador de esta tendencia en el país fue Pio Jaramillo Alvarado, su principal contribución fue su obra publicada en 1922: El Indio Ecuatoriano. Contribución al estudio de la sociología indoamericana. La posición de Jaramillo, junta la necesidad de incorporar al indio a la nación sin considerar lo que significa y vale su propia cultura, con el consejo de la "vuelta a la comunidad" o ayllu como la mejor forma de encontrar su unidad social. Además de su influencia en los estudios sociales, la literatura y el arte, ejerció importantes cargos públicos (diputado, senador y ministro de gobierno), que le permitió proponer soluciones en la organización sindical y en la legislación social ecuatoriana. Durante la década de 1930 a 1940, participó activamente en la aprobación de la legislación social que caracterizó a esa época.
En especial, durante la Asamblea Constitucional de 1937-1938 y en la dictadura del general Alberto Enríquez Gallo, en 1938, de tendencia izquierdista. Me refiero principalmente a la ley y estatuto jurídico que legalizó las comunas campesinas e indígenas, la ley de matrimonio civil y los códigos de menores y de trabajo. Toda esta legislación reconocía por primera vez una serie de derechos de los sectores populares ecuatorianos, especialmente de obreros, campesinos e indígenas. Es importante observar la participación de intelectuales mexicanos en estos avances, específicamente Vicente Lombardo Toledano, en el código del trabajo ${ }^{3}$ y, Moisés Sáenz en el tema indígena ${ }^{4}$.

En 1938, la Octava Conferencia Panamericana, realizada en Lima, sugiere enfrentar el problema en la región, a través de la confrontación de las experiencias existentes en cada país. A partir de esta iniciativa, el gobierno mexicano convoca al I Congreso Indigenista Interamericano que se realizó en Pátzcuaro (Michoacán) en 1940, con la presencia de dieciocho países del continente. La delegación ecuatoriana al mencionado Congreso estuvo presidida por Pío Jaramillo Alvarado.

El Congreso examina la situación del indio y toma varias decisiones al respecto. Una de las más importantes, fue la creación del Instituto Indigenista Interamericano y de Institutos en cada país. El primer director de esta nueva organización, hasta su muerte en 1960, fue el mexicano Manuel Gamio, quien logra que en 1948 se convierta en una agencia especializada de la Organización de Estados Americanos. Otra decisión significativa, fue una serie de medidas concretas para cambiar y abolir la situación de la población indígena, en especial, redistribución de la tierra, alfabetización y educación, saneamiento del medio ambiente, dignificación de la mujer, protección de la infancia, 
desarrollo de la agricultura y el artesanado y mejoramiento del régimen alimentario, habitacional y de trabajo.

Los planteamientos del indigenismo culturalista mexicano van a prevalecer en las nuevas políticas asumidas, reflejados en los tres grandes principios que fueron proclamados en el acto final del I Congreso: 1) El problema del indio es de interés público y reviste un carácter de urgencia; 2) El problema no es de índole racial sino de naturaleza cultural, social y económica y; por último, que 3) Los derechos de los indios deben ser protegidos y defendidos en el marco legal nacional y regional. Bajo estas consideraciones, el gobierno ecuatoriano creó en 1943 el Instituto Indigenista Ecuatoriano, cuyo primer director fue Jaramillo Alvarado. Sus objetivos fueron: "la investigación de la jurisprudencia a favor de los indios, la difusión de la "causa indígena" y la formación de expertos estatales en asuntos indígenas. Su órgano de difusión fue la revista Atahualpa (Moreno, 2006:29). Sin embargo, su permanencia no será perentoria debido a la falta de apoyo gubernamental, a diferencia de México que creó el Instituto Nacional Indigenista en 1948, bajo la dirección de Antonio Caso y que se estableció como agencia federal dotada de autonomía financiera y de personalidad jurídica y, permanece hasta la actualidad aunque con nuevo nombre a partir del 2003, se trata de la Comisión Nacional para el Desarrollo de los Pueblos Indígenas.

En un reciente artículo, Marc Becker (2007:136), refiriéndose al Instituto Indigenista Ecuatoriano ratifica su carácter de organización no indígena formado por un grupo de "médicos, economistas, sociólogos y abogados" mestizos, todos hombres de clase alta y media, que no pensaron ni preguntaron que querían los indígenas que para esa misma época formaban parte de un movimiento de lucha y resistencia que va a confluir en la creación de la Federación Ecuatoriana de Indios (FEI), en 1944, a propósito del Primer Congreso Ecuatoriano de Indígenas celebrado en Quito en agosto de ese año.

Becker critica las suposiciones de muchos académicos ecuatorianos y ecuatorianistas que consideran a la FEI como una instancia indigenista no estatal, encargada de mediar, expresar y traducción las verdaderas demandas indígenas. Este autor considera que si bien la FEI nace con el auspicio del Partido Comunista del Ecuador, también formaban parte de él importantes dirigentes indígenas, para él, "el Partido Comunista no formó el movimiento indígena, sino que los dos nacieron de la misma lucha" (Becker, 2007:139).

Para culminar con el período del pensamiento indigenista, es importante señalar que dentro de la relación entre la antropología mexicana y ecuatoriana, se aprecia la influencia del indigenismo culturalista mexicano en los intelectuales ecuatorianos, sin embargo, respecto a la influencia del indigenismo marxista, la comparación tiene otro carácter. Mientras en Ecuador este último combate frontalmente al primero, en México no sucede lo mismo. Tanto el proceso revolucionario de 1910 como la posterior reforma agraria, llevada a cabo por los presidentes Plutarco Elías Calles y Lázaro Cárdenas, no permitió más que una crítica demasiado tímida del uno al otro.

Otro elemento interesante, que posibilitó el pensamiento indigenista marxista, fue la introducción de temas de debate que aún ahora son motivo de discusión y disputa, se trata de la vigencia de la comunidad indígena como modelo de desarrollo propio y del reconocimiento de las nacionalidades indígenas, que ha sido 
incluido en las recientes constituciones boliviana y ecuatoriana, al definir a los respectivos Estados como plurinacionales.

\section{La práctica indigenista}

La práctica del pensamiento indigenista en América Latina tiene como distintivo esencial, el carácter aplicado de las propuestas teóricas hechas; en tanto se trataba de traducir a políticas públicas, programas, proyectos y acciones concretas del gran cúmulo de conocimientos generados durante las primeras cuatro décadas del siglo XX. Desde los inicios de la antropología como ciencia se planteó el tema de su aplicación, es decir, el uso de los de los resultados obtenidos a través del proceso investigativo en la resolución de problemas prácticos, ya sea suministrando información, proponiendo planes de acción o involucrándose en la acción directa.

Muchos de los representantes del indigenismo culturalista mexicano, como se mostró en el acápite anterior, tuvieron y mantuvieron sus vínculos académicos con la academia norteamericana. $A$ partir de esta segunda fase de desarrollo, México se va a convertir en el lugar preferido de trabajo de campo de muchos de ellos. Cabe mencionar el caso de Robert Redfield, que conoció a Gamio luego de la revolución de 1910, proveniente de la Universidad de Chicago, y de George M. Foster, de la Universidad de Berkeley en California ${ }^{5}$.

Ambos hicieron las primeras monografías etnográficas sobre el tema del campesinado, el primero con los trabajos clásicos hechos en Tepoztlán (Morelos) en 1930 y Chan Kom, en Yucatán, en 1934, y el segundo en Tzintzuntzan (Michoacán). Su interés por las sociedades campesinas estuvo unido al tema del cambio social, proveniente del proceso de urbanización de las sociedades complejas que de alguna manera preocupaba también a los pensadores del indigenismo culturalista.

En el Ecuador, el período de 1925 a 1960 se caracterizó por atravesar dos etapas claramente definidas. La primera, de 1925 a 1948, se inicia con la "revolución juliana", a través de un golpe de Estado de los mandos medios militares que rompió con el régimen oligárquico liberal, al cual le sucedieron veintidós cambios de gobierno entre presidentes, encargados del poder y dictadores. Además de la realización de cuatro asambleas nacionales, que pugnaron por poner las bases de una verdadera democracia en el país. Luego de la depresión económica mundial de 1930, surgen nuevos actores políticos en el país: los partidos de izquierda, los obreros, los campesinos y los indios acompañados de una importante jurisprudencia que les reconoció sus derechos como ciudadanos y ecuatorianos.

La segunda etapa, de 1948 a 1960, caracterizada por doce años de estabilidad política a través de tres gobiernos sucesivos luego de la pérdida de territorio; en 1941, por la confrontación armada con el Perú y, de la asamblea constituyente multiclasista de 1944, se inicia en el país un proceso de institucionalización del Estado caracterizado por una estabilidad económica temporal debido al auge bananero y, por la emergencia de políticas públicas sociales y culturales de mediano plazo. Mientras tanto, en México, la creación del Instituto Nacional Indigenista (INI) en 1948, bajo la dirección de Alfonso Caso, va a convertirse en el punto de referencia práctica en América Latina del indigenismo culturalista auspiciado y financiado por el Estado. En especial la creación de los centros coordinadores indigenistas, iniciados en 
Chiapas en 1951, una versión de misiones culturales dirigidos por un antropólogo más un equipo interdisciplinario de médicos, técnicos agropecuarios, educadores, abogados y comunicadores que incidieron en cinco grandes campos de intervención: salud, agricultura, educación, asuntos jurídicos y comunicación.

Un autor que tuvo mucha incidencia en México y en Ecuador, a través de sus discípulos, fue Gonzalo Aguirre Beltrán, un médico de profesión que luego estudió antropología. Su obra más relevante fue Regiones de Refugio publicada tardíamente en 1967 , la cual plantea la existencia de un proceso "dominical" de dependencia y dominio que une a los indígenas con los mestizos en aquellos espacios de relación social que son las "regiones de refugio".

En América del Sur, los países que no poseían los mismos recursos humanos y económicos del Estado mexicano, tuvieron que acudir a la cooperación multilateral para poner en práctica los acuerdos de política indigenista decididos en el I Congreso Indigenista Interamericano de 1940. Las Naciones Unidas en un esfuerzo interagencial ${ }^{6}$ con los gobiernos de Bolivia, Ecuador y Perú en primera instancia, y en una segunda, con Colombia, Chile, Argentina y Venezuela, pusieron en marcha la Misión Andina en 1953, concentrada en áreas de población indígena con un claro enfoque de desarrollo comunitario, como modelo de integración y modernización de los productores rurales de países en "vías de desarrollo".

La Misión Andina del Ecuador, iniciada en 1954 y cerrada en 1972, luego de ser nacionalizada en 1964, tuvo dos etapas de ejecución, una primera de proyectos pilotos en Otavalo, provincia de Imbabura y en el área cercana a Riobamba, provincia de Chimborazo, de muy pocos logros, según lo constató el evaluador del Instituto Indigenista Interamericano, el antropólogo español exiliado en México Juan Comas (Bretón, 2001), y una segunda, a partir de 1959, que incorporó a seis provincias más. Igual labor de evaluación cumplieron más adelante los antropólogos A. D. Marroquín, Alfonso Villa Rojas y el mismísimo Gonzalo Aguirre Beltrán. En este período, se inicia la relación de profesionales ecuatorianos con la escuela indigenista mexicana. Uno de los primeros fue Gonzalo Rubio Orbe, discípulo de Pío Jaramillo Alvarado, maestro de vocación y formación, autor en 1956, de una de las primeras etnografías sobre una comunidad indígena y mestiza cercana a la ciudad de Otavalo, denominada Punyaro. Estudio de Antropología Social y Cultural de una comunidad indígena y mestiza de Ecuador, basada en un trabajo de campo de seis meses de duración, con la utilización de los métodos investigativos desarrollados por la antropología. Aunque no realizó estudios antropológicos en México, recibió la influencia de los ideólogos del indigenismo, hasta el punto de convertirse en el director del Instituto Indigenista Interamericano de 1971 a 1977.

Alfredo Costales y Piedad Peñaherrera de Costales, formaron una pareja de investigadores preocupados del tema de la diversidad cultural del país. Aunque ninguno fue antropólogo de carrera, el primero hizo estudios de maestría en la Escuela Nacional de Antropología e Historia, ENAH, entre 1958 y 1962. Discípulos de Jaramillo Alvarado y del etnólogo francés Paul Rivet, fueron autores de una copiosa obra sobre tópicos indígenas, afroecuatorianos y mestizos. Alfredo Costales, fue el último director de la Misión Andina del Ecuador hasta su cierre en 1974. Los dos autores formaron parte del grupo fundador del Instituto Ecuatoriano de Antropología y Geografía (IEAG) creado en 1950, en sustitución 
del mencionado Instituto Indigenista Ecuatoriano iniciado en 1943, que también tuvo una vida corta al desaparecer en 1974.

Aníbal Buitrón es considerado el primer antropólogo ecuatoriano de carrera, en 1945 se doctoró en la Universidad de Chicago e inició junto con su esposa, Bárbara Salisbury, también antropóloga, investigaciones sobre la zona de Otavalo. Su obra más representativa fue la que escribió con su colega John Collier, Jr. en 1949, denominada El valle del amanecer, que fue publicada por la Universidad de Chicago. Buitrón fue funcionario y consultor internacional de Naciones Unidas por muchos años, e impulsor de la política de desarrollo de la comunidad, además de académico de algunas universidades norteamericanas.

Al cerrar este período, dos antropólogos ecuatorianos harán sus estudios en la Escuela Nacional de Antropología e Historia, se trata de Hugo Burgos y Gladys Villavicencio, que en sus respectivas obras, Relaciones interétnicas en Riobamba. Dominio y dependencia en una región indígena ecuatoriana (1970) y, Relaciones interétnicas en Otavalo-Ecuador. ¿Una nacionalidad india en formación? (1973), publicadas por el Instituto Indigenista Interamericano, intentan aplicar en el país las categorías propuestas por Aguirre Beltrán para el caso de México, sobre regiones de refugio, desarrollo de la comunidad y proceso dominical. A manera de conclusión, según Chávez:

\footnotetext{
"Las políticas indigenistas de los años sesenta y setenta, pusieron énfasis en dos aspectos: uno práctico, reflejado en programas de desarrollo regional, aplicados simultáneamente a nivel comunal; otro teórico, con el estudio de la naturaleza de las relaciones interétnicas que resalten las asimetrías originarias que afectaban a muchas de las comunidades indígenas" (2006:160-172).
}

Los estudios étnicos iniciados en esta época por la antropología ecuatoriana, se verán eclipsados por los estudios de inspiración marxista que van a dejar de lado el tema étnico, hasta que sean asumidos nuevamente por la emergencia política del movimiento indígena en los inicios de la década de los noventa, en un nuevo contexto y con influencias teóricas diferentes.

Una diferencia muy marcada con México en la etapa analizada, es que la antropología indigenista ecuatoriana, tanto culturalista como marxista, no tuvo el peso académico y político de la antropología mexicana indigenista, que marcó casi cuarenta años de debate y acciones prácticas. En Ecuador, aunque sí hubo pensadores indigenistas, el Estado no desarrolló una política pública e institucionalidad propia; apoyó el desarrollo de un programa con su financiamiento parcial conjuntamente con la cooperación internacional, pero no tuvo los recursos económicos ni la decisión política necesaria para mantenerla en el tiempo.

De tal manera, que no se puede encontrar en Ecuador en aquel período, instituciones estatales del tipo del Instituto Nacional Indigenista mexicano, ni instancias docentes e investigativas especializadas en la formación de antropólogos profesionales como la Escuela Nacional de Antropología e Historia ya mencionada, que permitieran establecer comparaciones entre las dos realidades con el fin de evaluar los aportes y limitaciones de esta corriente de pensamiento tan importante en América Latina.

Antes de continuar y, a modo de paréntesis, es importante señalar que el resto de los campos que forman parte del desarrollo de la antropología ecuatoriana, me refiero a la arqueología, la etnohistoria, los estudios sobre lengua y cultura y los pueblos indígenas amazónicos, cuyo avance 
fue paralelo a la antropología social, tuvieron muy poca relación con la antropología mexicana, los principales aportes y elaboraciones vendrán de influencias foráneas provenientes de Estados Unidos, Alemania y Francia.

Esto lo menciono porque a diferencia del indigenismo, que fue difundido a través de la relación sur-sur de antropologías latinoamericanas, en los otros campos de la antropología ecuatoriana no solo la influencia llegó desde el norte sino que también una gran parte de los antropólogos que investigaron en forma pionera estos campos eran extranjeros, son los ahora llamados "ecuatorianistas"7.

\section{La profesionalización e institucionalización de la antropología ecuatoriana}

En enero 1971, un grupo de once intelectuales, la mayoría latinoamericanos y todos no- indígenas, se reunieron en la isla caribeña de Barbados para considerar la relación entre las ciencias sociales y un tema que apenas emergía en el escenario político regional: "la liberación indígena". La declaración de Barbados I que resultó de esa reunión y, que posteriormente adquirió cierta fama, incluyó el siguiente párrafo sobre "la responsabilidad de la antropología":

"La antropología que se requiere ahora en América Latina no es lo que toma al indígena como objeto de estudio, sino, que percibe la situación colonial y se compromete a la lucha por la liberación. En este contexto, el rol de antropología es el de proveer a los pueblos colonizados los datos e interpretaciones, sobre ellos mismos y sus colonizadores, que serán de utilidad para sus luchas propias por la libertad..."
Esta afirmación, sencilla pero potente, trajo a colación una serie de preguntas claves para la antropología de la década de los setenta. ¿Por qué era necesario descolonizar la antropología latinoamericana? ¿De qué manera el pensamiento indigenista vigente en ese momento debía ser cuestionado fuertemente al ser otra forma de colonización antropológica? ¿Cómo plantear una antropología en la cual los "objetos" de investigación requerían pasar a ser "sujetos"? La experiencia de la segunda reunión de Barbados, celebrada en 1977, proporciona algunas pistas para comenzar a contestar estas preguntas. Después del éxito de la primera reunión, el siguiente paso lógico fue de sentar las bases para un diálogo entre los antropólogos solidarios y una selección de intelectuales indígenas que protagonizaban sus luchas. Según el relato de un antropólogo participante, la discusión sobre la relación entre antropología (o ciencias sociales en general) y los movimientos indígenas resultó sumamente polémica y, al fin, fue imposible llegar a acuerdos claros al respecto. Por ende, la Declaración asociada con esta segunda relación, elude el tema por completo para enfocarse exclusivamente en la necesidad de "organización política propia", que lucha en contra de la "dominación física y cultural". El inciso que más se acerca al tema de investigación sigue esta misma línea de desarrollo autónomo, enfatizando la necesidad de "(...) conservar y reforzar las formas de comunicación interna”, y de “(...) mantener los esquemas culturales básicos especialmente relacionados con la educación del propio grupo". Pareciera que los protagonistas indígenas de esa reunión no encontraron rol alguno para los antropólogos solidarios que, seis años, atrás se habían proclamado a favor de la "liberación indígena." 
Esta segunda reunión brinda un punto de partida para el presente acápite. La hipótesis que quisiera explorar aquí es que la relación entre los investigadores, antropólogos y los protagonistas de los movimientos sociales, en general, es más contradictoria, o al menos dotada de una tensión inevitable, que no ha recibido su debido reconocimiento. Lo anterior no quiere decir que la relación no sea fructífera, al contrario, la práctica de la investigación descolonizada tiene mucho que aportar, en cuanto a los resultados analíticos y, más aún, conocimientos que los movimientos mismos podrían aprovechar. Luego de treinta años de esta declaración, los resultados logrados muestran que han rendido mejor si se reconoce que se han logrado a través de tensiones inherentes entre la investigación y el protagonismo político y, no con la ilusión que es posible suprimir o superar tales tensiones.

Entre los participantes de las reuniones de Barbados se encontraba el antropólogo mexicano Guillermo Bonfil-Batalla, quien con un conjunto de colegas, publicaron en 1970 el manifiesto titulado De eso que Ilaman antropología mexicana, que marcó el relevo generacional de los antropólogos mexicanos y, el paso del indigenismo al denominado indianismo. Se trataba de una nueva versión del relativismo cultural que proclamaba a la aculturación como etnocidio y, por consiguiente, condujo a ontologizar la indianidad y absolutizar la diferencia entre la población indígena con la no-indígena. El Estado mexicano se ve precisado a pasar de una política integracionista respecto a los pueblos indígenas, hacia una política de gestión étnica, iniciando de esta manera una nueva etapa de la antropología latinoamericana.

Tanto México como Ecuador, como países, van a enfrentar en esta etapa un conjunto de cambios sociales claves; la rebelión estudiantil de 1968 remeció el duro aparato estatal en poder del PRI mexicano desde 1930, las leyes de reforma agraria de 1964 y 1973 en Ecuador van a terminar con las relaciones de trabajo obligatorio en la Sierra y a permitir el acceso a la tierra a los pequeños productores en la Costa.

La crisis social se ve agravada por la crisis financiera y económica y, paralelamente, se da comienzo al establecimiento del modelo económico neoliberal impulsado por el Fondo Monetario Internacional, que va a conducir al abandono del agotado modelo de industrialización sustitutiva de importaciones y lo va a reemplazar por las leyes del libre mercado. El Estado empieza un proceso de desconcentración, descentralización y regionalización de los servicios públicos a la par que inicia un agresivo proceso de privatización de los mismos. La crisis económica también llega acompañada por un sistemático proceso de endeudamiento internacional con la banca bilateral y privada cuyo pago va a acaparar un porcentaje significativo de las divisas nacionales. En este contexto complejo, hay dos temas que la antropología mexicana va a desarrollar: los estudios de diversidad cultural y étnica y, los relacionados con la etnohistoria mesoamericana y andina que le van a vincular con la antropología ecuatoriana. Cada uno de ellos requiere un tratamiento por separado, sin embargo, la relación que se establece entre ambas antropologías tiene un doble vínculo a través de la formación de profesionales ecuatorianos en México desde finales de los sesenta y, de la creación del primer departamento de antropología en la Pontificia Universidad Católica del Ecuador en 1971.

Durante la década de los sesenta y setenta, dos generaciones de antropólogos ecuatorianos van a realizar estudios de pregrado y posgrado en la 
Universidad Iberoamericana y en el recientemente creado (1973) Centro de Investigaciones Superior del Instituto Nacional de Antropología e Historia (CISINAH), hoy Centro de Investigaciones y Estudios Superiores en Antropología Social (CIESAS). La figura central de estas dos instituciones fue el antropólogo Angel Palerm Vich, refugiado español nacionalizado mexicano, que junto con otros profesores también expulsados de sus instituciones por haber participado en el movimiento estudiantil de 1968, revolucionaron la enseñanza de la antropología social mexicana, con la interesante combinación de profesores y alumnos provenientes de la propia lberoamericana, de la ENAH y de diversos centros de formación eclesiástica (sobre todo jesuitas), que por entonces experimentaban tiempos de revisión y renovación bajo la influencia de la Teología de la Liberación.

De regreso al país, los antropólogos formados en México junto con otros ecuatorianos formados en los Estados Unidos, Francia y Alemania, ponen en marcha el departamento de antropología de la Universidad Católica, que a partir de 1977 va a graduar a los primeros antropólogos formados en el país y profesionalizará la disciplina.

Retomando el tema de los estudios de la diversidad cultural y étnica mexicanos, planteados desde el marxismo y la antropología política de la época, a la luz de las teorías de A. V. Chayanov, Eric R. Wolf y Sidney Mintz, se inicia un largo siglo de los denominados estudios campesinos, con la especificidad que Palerm y sus seguidores van a introducir en sus investigaciones la relación estrecha entre la antropología y la historia por un lado, y por otro, la práctica investigativa basada en largas temporadas de trabajo de campo para luego hacer ciencia.
Si se revisa los títulos de las tesis de los primeros estudiantes graduados en el Departamento de Antropología de la Universidad Católica, el tema de la economía y política campesina es reiterativo. Este interés en los dos países va a derivar más adelante en el retorno a los estudios étnicos con énfasis en el tema indígena, y luego, en los demás grupos diversos que conforman ambos países: afro descendientes, minorías étnicas, etc.

El campo de los estudios etnohistóricos andinos y mesoamericanos, va a ser impulsado en forma simultánea desde la academia norteamericana y latinoamericana. Mediante la propuesta de pensadores marxistas que recogen la discusión de la formación del Estado y, en especial, de las sociedades hidráulicas, tanto la civilización maya y azteca y la civilización inca, me refiero a Karl Wittfogel, Stanley Diamond, William Sanders, Pedro Carrasco, Richard Schaedel y John Murra, que la juntaron con la etnografía de las sociedades campesinas e indígenas contemporáneas. En realidad, tanto Palerm desde México a través del CISINAH, como Murra ${ }^{8}$ desde los Andes a través del Instituto de Estudios Andinos, se van a convertir en los animadores de la investigación etnohistórica de ambas realidades; la amistad que unió a estos dos autores, que se conocieron en los Estados Unidos, dio como resultado la formación de grupos de investigadores locales que harán importantes contribuciones a la antropología de ambos países. Además estos dos investigadores inauguraron un ejercicio que ha sido poco desarrollado entre las antropologías del sur, el ejercicio comparativo, que constituye un método de larga data en la antropología, el que desgraciadamente no ha tenido continuidad a través del tiempo y que en la actualidad se vuelve indispensable para el desarrollo de la disciplina. 
Para finalizar, caben algunas reflexiones para contestar la pregunta planteada en el título de este trabajo, la relación entre la antropología mexicana y ecuatoriana: ¿un camino de ida y vuelta? La respuesta es definitivamente sí, el recorrido hecho durante seis décadas de relación lo demuestra justificadamente.

En el período de la propuesta indigenista la relación se centró más en la práctica que en la teoría, las demandas internacionales sobre el tema que plantearon las resoluciones del I Congreso Indigenista Interamericano, llevaron a privilegiar este tipo de relación. La presencia de antropólogos mexicanos en Ecuador abrió la posibilidad para que antropólogos ecuatorianos se formaran en los centros académicos de ese país, y luego de su retorno iniciaran de manera profesional la carrera de antropología, lo que permitió su institucionalización. El balance más importante en esta ida y vuelta, visto a la distancia, es desde el lado mexicano, los temas de investigación y debate que plantearon en su momento a la antropología latinoamericana en general, el interés por los estudios de la diversidad cultural y étnica, y el énfasis en la relación de la antropología con la historia.

Desde el lado ecuatoriano, el aporte de intercambio viene principalmente por la riqueza y dinámica de la problemática étnica y cultural del país y, también, por el esfuerzo de plantear la importancia de los estudios comparativos entre los Andes y Mesoamérica, como una forma de referencia y aprendizaje mutuo de realidades que aunque específicas enfrentan problemas comunes.

\section{Notas}

${ }^{1}$ A la par de las investigaciones de Boas, Bronislaw Malinowski desarrollaba su propia tradición de trabajo de campo en las lejanas islas Trobriand del Pacífico
Occidental, privilegiando más al observador que al observado.

${ }^{2}$ Me refiero al concepto de "aculturación", planteado por Aguirre Beltrán (1970) en 1958, quien fue discípulo de Gamio y Caso.

${ }^{3}$ Vicente Lombardo Toledano, discípulo de Antonio Caso aunque luego rompió con él, creó la Confederación de Trabajadores de México (CTM), en 1936 y en 1938 fue director de la Confederación de Trabajadores de América Latina (CTAL).

${ }^{4}$ Moisés Sáenz, discípulo de Gamio, fundó en 1932 la primera Estación Experimental de Incorporación del Indio en Carapán, entre los Tarascos de Michoacán, antecedente de los futuros Centros Coordinadores del Instituto Nacional Indigenista. Entre enero de 1934 y enero de 1935 fue embajador de México en Ecuador, lo que le sirvió para conocer el país y escribir el libro Sobre el indio ecuatoriano y su incorporación al medio nacional, publicado en México por la Secretaría de Educación Pública en 1943.

${ }^{5}$ En el caso del Perú, la relación entre indigenistas peruanos y antropólogos norteamericanos de la Universidad de Cornell puede ser analizada en el proyecto Vicos, iniciado en los Andes Peruanos en 1951, con el fin de producir el cambio social y su respectiva ingeniería en condiciones de "laboratorio".

${ }^{6}$ Las agencias que intervinieron fueron la Organización Internacional del Trabajo (OIT), la Organización para la Alimentación y la Agricultura (FAO), la Organización Mundial de la Salud (OMS), la Organización para la Educación, la Ciencia y la Cultura, (UNESCO), el Fondo Internacional para la Infancia (UNICEF), y el Programa Mundial de Alimentos (PAM).

${ }^{7}$ Para mayor detalle ver los trabajos de Moreno 2006 y Martínez 2007.

${ }^{8}$ El primer contacto de Murra con los Andes fue en el año 1941, cuando llego al Ecuador a hacer una investigación arqueológica sobre la presencia de la cultura Chavín en los Andes del norte, como parte del proyecto de Donald Collier, profesor de la Universidad de Chicago (Castro, Aldunate e Hidalgo, 2000). 


\section{Referencias bibliográficas}

Aguirre Beltrán, G. (1970). El proceso de aculturación y el cambio sociocultural en México. México: Editorial Comunidad, Universidad Iberoamericana.

Bonfil Batalla, G., et.al. (1970). De eso que llaman antropología mexicana. México: ENAH.

Becker, M. (2007). "Comunistas e indigenistas en la formación del movimiento indígena”, en Iconos, № 27, pp.135-144.

Cardoso de Oliveira, F. (1998). "Antropologias periféricas versus antropologias centrais". En O trabalho do antropólogo, editado por Roberto Cardoso de Oliveira, 107-133. Sao Paulo: Editora UNESP.

Castro, V., Aldunate, C. \& Hidalgo, J. (eds.) (2000). Nispa Ninchis. Conversaciones con John Murra. Lima: Instituto de Estudios Peruanos e Instituto de Estudios Andinos.

Chávez, G. (2006). "A los 35 años de Relaciones Interétnicas en Riobamba". En Ecuador Debate, № 67, CAAP, pp.:160-172.

Escobar, A. (1996). La invención del tercer mundo. Bogotá: Editorial Norma.

Geertz, C. (1988). La Interpretación de las Culturas. Barcelona: Editorial Gedisa.

Jaramillo Alvarado, P. (1983). El Indio Ecuatoriano. Contribución al estudio de la sociología indo-americana. Quito: Corporación Editora Nacional.

Jimeno, M. (2007). "Naciocentrismo: tensiones y configuración de estilos en la antropología sociocultural colombiana". En Revista Colombiana de Antropología, vol. 43, enero-diciembre, pp. 9-32.

Krotz, E. (1997). "Anthropologies of the South: Their Rise, their Silencing, their characteristics". En Critique of Anthropology 17(3):237-251.

Martínez, C. (2007). "Antropología indigenista en el Ecuador desde la década de 1970: compromisos políticos, religiosos y tecnocráticos". En Revista Colombiana de Antropología, vol. 43, enero-diciembre, pp. 335-366.

Moreno, S. (2006). Pensamiento antropológico ecuatoriano. 2 tomos. Quito: Banco Central del Ecuador y Corporación Editora Nacional.
Ramos, A. R. (2007). “¿Hay lugar para el trabajo etnográfico?" En Revista Colombiana de Antropología, vol. 43, enero-diciembre, pp. 231-61.

Rappaport, J. (2007). "Más allá de la escritura: la epistemología de la etnografía en colaboración". En Revista Colombiana de Antropología, vol. 43, enero-diciembre, pp. 197-229.

Ribeiro, G. L. \& Escobar, A. (eds.) (2006). World Anthropologies. Disciplinary transformations within systems of power. Oxford New York: Editorial Berg.

Roig, A. A. (1979). "Los comienzos del pensamiento social y los orígenes de la sociología en el Ecuador". En Espinosa Tamayo, A. (1979). Psicología y sociología del pueblo ecuatoriano. Quito: Banco Central del Ecuador y Corporación Editora Nacional, pp. 9-127. 


\title{
CIENTO CUATRO AÑOS DE ANTROPOLOGÍA MEXICANA
}

\author{
One hundred and four years of Mexican Anthropology
}

\section{LUIS VÁZQUEZ LEÓN *}

\section{Resumen}

Luego de un siglo de existencia institucional y profesional la antropología mexicana ha constituido una treintena de escuelas, facultades, institutos, colegios y centros de investigación, todos los cuales ofrecen estudios de licenciatura, maestría y doctorado. En apariencia, es una disciplina consolidada. Es preocupante, sin embargo, que mientras prevalece el desempleo de los jóvenes profesionales, la política científica haya tenido el efecto de crear una élite menos interesada en los "grandes problemas nacionales" que en reproducir más profesionales con futuro incierto. La supuesta "comunidad antropológica" está escindida en una antropología académica y una antropología gubernamental, y los esfuerzos por reunirlas son aún incipientes. La élite está preocupada también por su visibilidad internacional, ubicando a la antropología gubernamental (que en su época de oro dio fama internacional a la antropología mexicana) en un nivel inferior, pese a su producción constante de estudios, los que también contribuyen al desarrollo disciplinar.

Palabras Clave: Instituciones antropológicas, Mundialización, Ciencia normal, Conflictos.

\section{Abstract}

After a century of institutional and professional existence, Mexican anthropology is formed by thirty schools, colleges, institutes, and research centers, all of which offer undergraduate, master's and doctoral degrees. Apparently, it is a consolidated discipline. It is disturbing, however, that while unemployment prevails among young professionals, science policies have had the effect of creating an elite less interested in the "larger national issues", than in the production of professionals with an uncertain future. The alleged "anthropological community" is divided into an academic anthropology and a governmental anthropology and the efforts to bring them together are still incipient. The elite is concerned about its international visibility, placing governmental anthropology (which in its golden age gave international fame to Mexican anthropology) at a lower level, despite its constant production of studies, which also contribute to the development of the discipline.

Key words: Anthropological institutions, Globalization, Normal science, Conflict

\footnotetext{
* Antropólogo. Doctor en Antropología Social. Investigador Centro de Investigaciones y Estudios Superiores en Antropología Social - CIESAS de occidente. Correo electrónico: Ivazquez@ciesas.edu.mx, Ivazquez@ciesasoccidente. edu. $\mathrm{mx}$
} 